\title{
Two-dimensional late-stage coarsening for nucleation and growth at high-area fractions
}

\author{
Norio Akaiwa* and D. I. Meiron \\ Applied Mathematics, 217-50, California Institute of Technology, Pasadena, California 91125
}

(Received 4 April 1996)

\begin{abstract}
Numerical simulations of two-dimensional late-stage coarsening for nucleation and growth (Ostwald ripening) are performed at large-area fractions without shape restrictions. We employ efficient computational methods that allow us to study large systems. The free energy of the system we consider is composed of two different curves. Thus, the system consists of a set of isolated particles even at high-area fractions. This is totally different from the interconnected spinodal structures generated by the Cahn-Hilliard model, where the free energy is composed of a single curve. Although the domain structures are quite different, we find that the qualitative features of the structure function for both models are the same. [S1063-651X(96)51207-9]

PACS number(s): 64.60.My, 64.60.Qb, 64.75+g
\end{abstract}

Phase separation of two phase mixtures has been observed in a variety of systems. When the structures of the two phases are the same, e.g., liquid-liquid mixtures or solidsolid mixtures with the same lattice structures, the CahnHilliard $(\mathrm{CH})$ model is generally used for theoretical and numerical studies [1]. In this case, the free energy of the system is composed of a single curve with two minima. The $\mathrm{CH}$ model generates interconnected spinodal structures near the critical composition, while near the coexistence line the system consists of a set of isolated particles that are generated by nucleation [2-6]. Between those two regions, morphological transition from the spinodal-like structures to a set of isolated particles occurs.

On the other hand, when the structures of the two phases are different, e.g., liquid-solid mixtures or solid-solid mixtures with different lattice structures, the free energy of the system is composed of two different curves. In this case, isolated particles are formed by nucleation at any quench position, so that spinodal decomposition does not occur. After the nucleation stage the particles grow in supersaturated solutions. When the supersaturation becomes small or the volume fraction of the particle phase becomes almost constant, large particles grow at the expense of small particles. This process is known as coarsening, or Ostwald ripening, and the steady-state diffusion equation with a GibbsThomson boundary condition is generally used for theoretical and numerical studies.

For both cases, it has been widely believed that the characteristic length $l_{c}(t)$ grows as $t^{1 / 3}$ in the late stage, which is the Lifshitz-Slyozov scaling law [7]. In addition, the structure function $\mathcal{S}(k, t)$ satisfies the scaling law $\mathcal{S}(k, t)=l_{c}^{d}(t) S\left(k l_{c}\right)$, where $k$ is the wave number, $d$ is the dimension of the system, and $S\left(k l_{c}\right)$ is the scaled structure function.

In this paper, we study the late stage of nucleation and growth in two dimensions using a boundary integral formulation for the steady-state diffusion equation without shape restrictions. In a previous paper [8], we performed numerical

\footnotetext{
*Present address: Division of Computational Materials Science, National Research Institute for Metals, 1-2-1 Sengen, Tsukuba 305, Japan.
}

simulations using a circular approximation. At high-area fractions, it is expected that the particles are no longer circular, since the diffusion field around a particle is highly asymmetric. Simulations without the shape restrictions have been performed using a small to moderate number of particles [9-11]. However, large-scale simulations have not been performed due to computational difficulties. We employ a fast method for solving iteratively the large matrices that result and a stable time integration method that enables us to consider very large systems.

The system we consider consists of $N$ particles in a square unit cell. Mass diffusion outside the particles is assumed to be the only mass transfer process. The dimensionless concentration $u$ outside the particles satisfies the steady-state diffusion equation

$$
\nabla^{2} u=0
$$

with a Gibbs-Thomson boundary condition

$$
u(\mathbf{p})=\kappa(\mathbf{p}) \text { for } \mathbf{p} \text { on } L^{j}
$$

where $\mathbf{p}$ is the position vector of the point $p, \kappa(\mathbf{p})$ is the curvature at $\mathbf{p}$, and $L^{j}$ is the interface of particle $j$. The total mass is conserved in the system, so that

$$
\sum_{j=1}^{N} \int_{L^{j}} \frac{\partial u(\mathbf{p})}{\partial n_{p}} d p=0
$$

holds, where the normal vector $\mathbf{n}_{p}$ is directed outward from the particle. The evolution equation of the interface $j$ is given by

$$
V^{j}(\mathbf{p})=\frac{\partial u(\mathbf{p})}{\partial n_{p}},
$$

where $V^{j}$ is the interfacial velocity.

In order to perform large-scale numerical simulations, very large matrices resulting from discretization must be solved. In addition, a very small time step must be used to avoid numerical instability due to the existence of the curvature term. These simulations cannot be performed on present day computers without using efficient computational meth- 
ods. Here we briefly describe the computational methods used in this paper. The details will be published elsewhere.

We seek a solution of Eqs. (1)-(3) as a sum of the potentials generated by a double layer density $\mu$ on each interface, sources $A^{j}$ located at the centers of mass $\mathbf{c}^{j}$, and an overall constant $u_{0}$ [12]. Since the double-layer potential is discontinuous across the boundary, we obtain the following integral equation of the second kind for $\mu$,

$$
\begin{aligned}
\kappa(\mathbf{p})= & -\frac{1}{2} \mu(\mathbf{p})+\frac{1}{2 \pi} \sum_{j=1}^{N} \int_{L^{j}} \mu(\mathbf{q}) \frac{\partial \ln |\mathbf{p}-\mathbf{q}|}{\partial n_{q}} d q \\
& +\sum_{j=1}^{N} A^{j} \ln \left|\mathbf{p}-\mathbf{c}^{j}\right|+u_{0} .
\end{aligned}
$$

Equation (5) has a unique solution under the following constraints,

$$
\begin{gathered}
\sum_{j=1}^{N} A^{j}=0, \\
\int_{L^{j}} \mu(\mathbf{q}) d q=0 \text { for } j=1, \ldots, N-1, \\
u_{0}=\frac{1}{2 \pi} \int_{L^{j}} \mu(\mathbf{q}) d q, \text { for } j=N .
\end{gathered}
$$

For numerical purposes, the integrals in Eqs. (5), (7), and (8) are discretized so that each interface is composed of markers equally spaced in arc length. In practice, 16-1024 markers are used to describe each interface depending on the size and the nearest distance from the other interfaces. Using conventional methods of evaluation of the integrals and solution of the resulting matrix equations, numerical simulations are limited to a small number of particles.

Since the diffusion field is screened due to the mass conservation constraint, the effects of other particles separated by a certain interaction distance can be neglected when computing the diffusion field around a given particle. In practice, the main computational cell is divided into square subcells that contain 50-150 particles, and only particles located within 6-7 times the average radius from the edge of the subcell are taken into account as interacting particles. The relative error introduced by this method decreases exponentially as the interaction distance increases [8], and the error due to this choice of the interaction distance is generally better than $1 \%$.

We use an iterative scheme, generalized minimal residual method (GMRES) [13], with a preconditioner [12] to solve the resulting matrix system described by Eqs. (5)-(8). This method requires one matrix-vector multiplication per iteration. The fast multipole summation method [14] is employed to evaluate each matrix-vector multiplication. Using this method, the computational cost for the matrix-vector multiplication is proportional to the size of the matrix rather than the square of the matrix size as in a direct method. In addition, it is unnecessary to store the large matrix in memory. Typically, the size of the matrix for one subcell is $10000-$ 25000 , and $20-25$ iterations are necessary to obtain a rela- tive residual error less than $10^{-8}$. It should be noted that the above method is about 2500 times faster than the direct method [12].

Once $\mu$ and the source strengths $A^{j}$ are computed, the interfacial velocity is calculated by computing the normal derivative of the potential on the interface. If an explicit Euler method is used for time integration, this results in a strong time-step constraint $\Delta t<B h^{3}$, where $h$ is a marker spacing, and $B$ is a constant. We remove this numerical stiffness using a method developed by Hou, Lowengrub, and Shelly [15]. Instead of using $x$ and $y$ coordinates on the interface, the particle shape is represented using the length of the interface $l$ and the angle $\theta$ between the tangential vector at the interface and $x$ axis. The evolution equations for $\theta$ and $l$ are then given by

$$
\begin{gathered}
\frac{\partial \theta^{j}(\alpha)}{\partial t}=\frac{2 \pi}{l^{j}}\left[-\frac{\partial V^{j}(\alpha)}{\partial \alpha}+\frac{\partial \theta^{j}(\alpha)}{\partial \alpha} T^{j}(\alpha)\right], \\
\frac{\partial l^{j}}{\partial t}=\int_{0}^{2 \pi} \frac{\partial \theta^{j}(\alpha)}{\partial \alpha} V^{j}(\alpha) d \alpha .
\end{gathered}
$$

Here, $\alpha$ is a parameter varying from 0 to $2 \pi$ on the interface, and $T^{j}$ is the tangential velocity along the interface which is introduced to keep the marker space constant. Hou, Lowengrub, and Shelly [15] extracted the stiffest part of Eq. (9) and showed that this contribution can be linearized in Fourier space,

$$
\frac{\partial \hat{\theta}^{j}(k)}{\partial t}=-\frac{1}{2}\left(\frac{2 \pi}{l^{j}}\right)^{3} k^{3} \hat{\theta}^{j}(k)+\hat{R}^{j}(k),
$$

where the hats refer to Fourier transform in space. The first term on the right-hand side of Eq. (11) is the stiff contribution, and $R$ is the remaining nonlinear term, which is less stiff than the first term. Since the first term represents a linear operator, an implicit Crank-Nicholson method can be applied with an explicit Euler method applied to the second part. Before updating $\theta, l$ is updated using the explicit Euler method, so that $\theta$ can be updated explicitly. In addition to $\theta$ and $l$ for a given interface, we need information about the evolution of the center of mass, which we use as a reference point for the particles. The interface is updated in $x, y$ space using the Euler method, and the new center of mass is computed for this configuration. In these simulations we choose the time step $\Delta t=0.0025\langle r\rangle^{3} /\left\langle r_{0}\right\rangle^{3}$, where $\langle r\rangle$ is the average radius, and $\left\langle r_{0}\right\rangle$ is the initial average radius chosen to be 1 in the simulations. It should be noted that if the explicit Euler method is used for time integration of $\theta$, the time step has to be 1000 times smaller in order to obtain a stable time integration.

We use initial configurations with 5000 particles. These are generated by simulations using the monopole and dipole approximations starting from 100000 particles [8]. The purpose of these initial rough simulations using the monopole and dipole approximations is to obtain particle configurations close to those in the scaling regime. During the simulations, particles smaller than $0.05\langle r\rangle$ are removed from the system. The simulations are continued until the number of particles drops to 50. Four independent runs are performed at area 


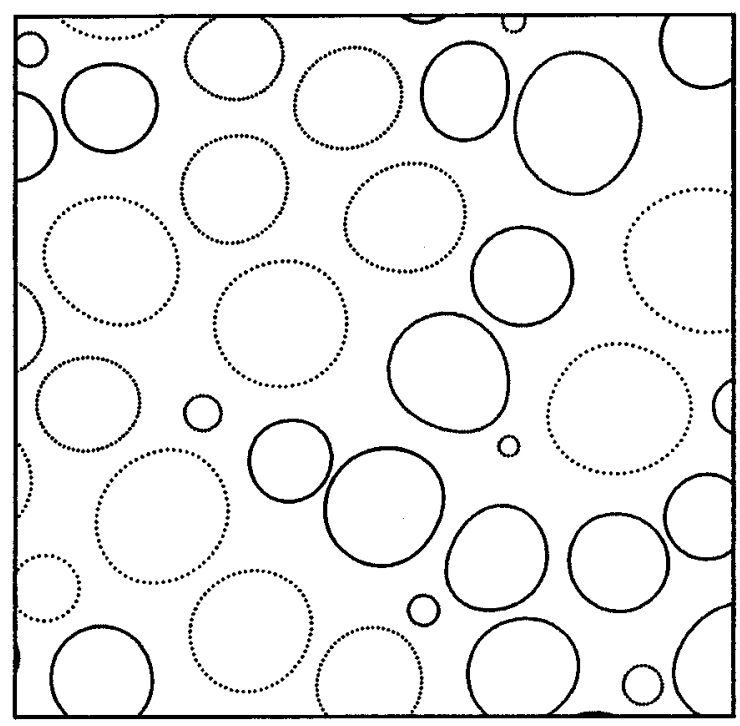

FIG. 1. Particle configuration in the late stage at $\phi=0.5$. Only a part of the 171-particle system is shown. The size of the box is $15\langle r\rangle$.

fraction $\phi=0.4$ and three runs are performed at $\phi=0.5$. The results shown are the averages of these runs.

We define the radius of a noncircular particle as $r_{j}=\sqrt{a_{j} / \pi}$, where $a_{j}$ is the area of the particle $j$. In order to investigate the coarsening, we compute $\langle r\rangle$ versus $t$ and fit this to a power law of the form $\langle r\rangle \sim t^{n}$. The exponent $n$ is determined by a linear regression of a $\log -\log$ plot of $\langle r\rangle$ versus $t$ in the late stage. We define the late stage as $t\rangle 20$, where the number of particles still remaining in the system is about 500 . We found that the exponents in the late stage are $n=0.324 \pm 0.008$ and $n=0.326 \pm 0.005$ at $\phi=0.4$ and 0.5 , respectively. These values are close to the expected value of $1 / 3$.

Figure 1 shows a snapshot of the coarsening system with $\phi=0.5$ at $t=128$. Only a part of the 171-particle system is shown in the figure, and the size of the box shown is $15\langle r\rangle$. It is interesting to note that interconnected spinodal structures are generated by the $\mathrm{CH}$ model at this area fraction [2-4]. The non-circularity of the particles is clear in the figure, and a very similar morphology is observed in experiments [16].

To estimate the degree of noncircularity, we define a shape factor for a given particle $j$ as $P^{j}=l^{j} / 2 \pi r_{j} . P$ is one for a circle, and $P>1$ implies noncircular particles. The average shape factor is plotted as a function of time in Fig. 2. In the present simulations, all particles are initially circular. As seen in Fig. 2, $\langle P\rangle$ exhibits an overshoot and then appears to asymptote to a constant value. Similar results are shown in the work of Rogers and Desai [3] in their simulations using the $\mathrm{CH}$ model at off-critical compositions. In this case, a shape transition occurs from the spinodal-like structure to a set of isolated noncircular particles. Although the shape factor is time dependent in their simulations, the shape factor seems to approach a constant value. It should be noted that the shape transition does not occur at the critical composition, since the $\mathrm{CH}$ model is symmetric at this composition.

We define the structure function $\mathcal{S}(k)$ as

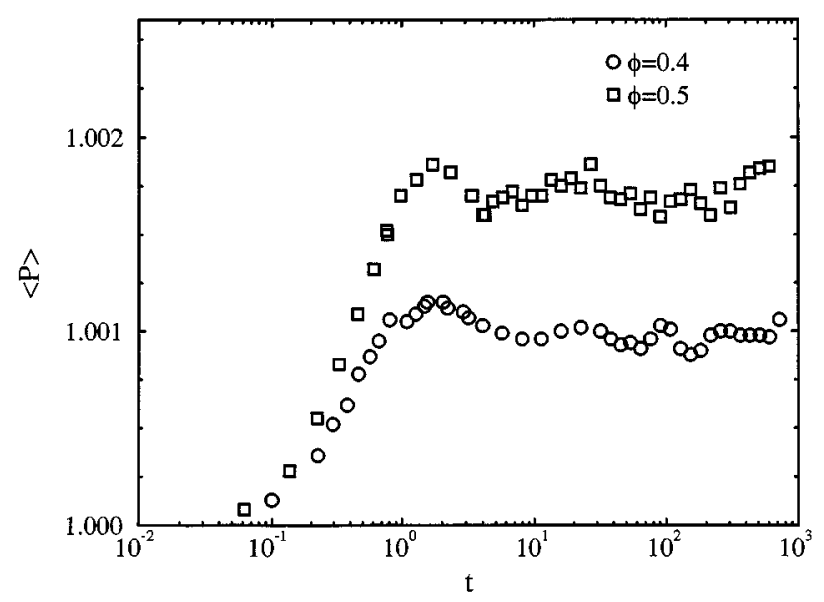

FIG. 2. Average shape factor $\langle P\rangle$ as a function of time.

$$
\mathcal{S}(k)=\langle\mathcal{S}(\mathbf{k}) \mathcal{S}(-\mathbf{k})\rangle_{\Omega},
$$

where \langle\rangle$_{\Omega}$ is defined as a circular average, and

$$
\mathcal{S}(\mathbf{k})=\sum_{j}^{N} \int_{a^{j}} \exp (i \mathbf{k} \cdot \mathbf{x}) d x .
$$

We also define a normalized structure function $s(q)$ which is normalized so that $q^{3} s(q)=1$ as $q \rightarrow \infty$, where $q=k\langle r\rangle$. Figure 3 shows a $\log -\log$ plot of the normalized structure function in the late stage. The results shown are the average of results from 11 different times for $t>128$. For $q>0.5$, the structure functions at earlier times $(20<t<128)$ are indistinguishable from those shown in Fig. 3. Only the small-wavenumber portion of $s(q)$ (for $q<0.5)$ is time dependent. If the results for $s(q)$ at different times are plotted, we observe that the small $q$ portion is approaching a straight line of slope 4 as time increases. Thus, it is likely that $s(q)$ varies as $q^{4}$ at small $q$ in the late stage [17-20]. This is the most difficult part to obtain numerically. Tomita [19] showed that this $q^{4}$ behavior is due to the rotational symmetry of the system. He also showed that the coefficient of the contribution varying

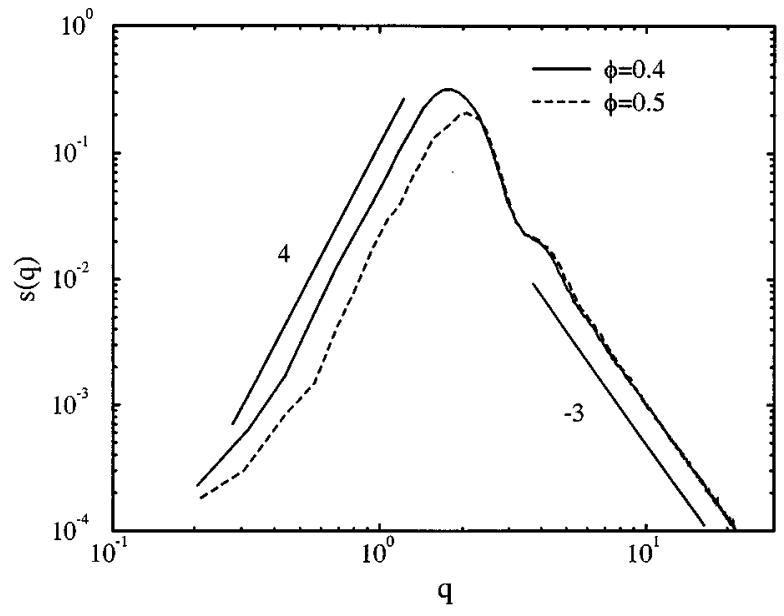

FIG. 3. A log-log plot of the normalized structure function in the late stage. The solid lines indicate slopes of 4 and -3 , respectively. 
with $q^{2}$ is a slowly decreasing function of time and related to the asymmetry of the system. At large $q, s(q)$ has an asymptotic form of $s(q)=q^{-3}$. This is the Porod law, which originates from the sharp interface. The interface has no thickness in the present model, so the Porod law is always satisfied. A shoulder at $q=3 \sim 4$ is clearly observed in Fig. 3. For systems that consist of isolated particles, this behavior is explained by the polydispersity of the particles [8,20]. All of the above qualitative features are also observed in simulations of spinodal decomposition [20]. Although the domain structures in the present simulations are quite different from the spinodal structures, all of the qualitative features in the structure function are the same.

If the structure function using the circular approximation [8] is plotted along with the present results at $\phi=0.4$, the two results are almost identical. In addition, the particle size distribution functions for circular and noncircular simulations are very close at this area fraction. Thus, we can conclude that the circular approximation is valid even at $\phi=0.4$. Although the shape of particles is not circular, the statistical properties of the system are not largely affected by the noncircular shapes at this area fraction. We did not perform simulations using the circular approximation at $\phi=0.5$ since we found serious particle overlaps.

The present simulations can be extended to higher-area fractions. However, the simulations become difficult as the area fraction increases. Occasionally we found that the separation distance between two interfaces becomes smaller than the marker spacing. In this case we reposition the particles so that their separation is at least two times larger than the marker spacing; otherwise the error in the normal velocity becomes large and the time integration leads to interfaces that intersect. These events are rare at the present area fractions, but the number of such events may increase as the area fraction increases. We have not yet confirmed that particle overlap can be avoided by reducing the time step and increasing the number of markers for those particles in close proximity to one another.

We thank Professor P. W. Voorhees for helpful comments. This work was supported by DOE Grant No. DEFG03-89ER-25073.
[1] A. J. Bray, Adv. Phys. 43, 357 (1994).

[2] Y. Oono and S. Puri, Phys. Rev. A 38, 434 (1988).

[3] T. M. Rogers and R. C. Desai, Phys. Rev. B 39, 11956 (1989).

[4] A. Chakrabarti, R. Toral, and J. D. Gunton, Phys. Rev. E 47, 3025 (1993).

[5] A. M. Lacasta, J. M. Sancho, A. Hernández-Machado, and R. Toral, Phys. Rev. B 48, 6854 (1993).

[6] N. Masbaum, J. Phys. (France) I 5, 1143 (1995).

[7] I. M. Lifshitz and V. V. Slyozov, J. Phys. Chem. Solids 19, 35 (1961).

[8] N. Akaiwa and D. I. Meiron, Phys. Rev. E 51, 5408 (1995).

[9] P. W. Voorhees, G. B. McFadden, R. F. Boisvert, and D. I. Meiron, Acta Metall. 36, 207 (1988).

[10] T. Imaeda and K. Kawasaki, Physica A 164, 335 (1990).

[11] J. Zhu, X. Chen, and T. Y. Hou (unpublished).
[12] A. Greenbaum, L. Greengard, and G. B. McFadden, J. Comput. Phys. 105, 267 (1993).

[13] Y. Saad and M. H. Schultz, SIAM J. Sci. Stat. Comput. 7, 856 (1986).

[14] L. Greengard and V. Rokhlin, J. Comput. Phys. 73, 325 (1987).

[15] T. Y. Hou, J. S. Lowengrub, and M. J. Shelly, J. Comput. Phys. 114, 312 (1994).

[16] O. Krichevsky and J. Stavans, Phys. Rev. E 52, 1818 (1995).

[17] C. Yeung, Phys. Rev. Lett. 61, 1135 (1988).

[18] H. Furukawa, J. Phys. Soc. Jpn. 58, 216 (1989).

[19] H. Tomita, Prog. Theor. Phys. 85, 47 (1991).

[20] P. Fratzl, J. L. Lebowitz, O. Penrose, and J. Amar, Phys. Rev. B 44, 4794 (1991). 